\title{
Chylous ascites complicating anterior resection for colorectal cancer
}

A 76-year-old female underwent an anterior resection for adenocarcinoma in the upper third of the rectum. Preoperative staging demonstrated no radiological evidence of metastatic disease. Postoperative recovery was uncomplicated. The histology demonstrated a T3 N0 $44 \mathrm{~mm}$ mucinous adenocarcinoma that had been completely excised. There was no evidence of metastatic disease seen within 31 lymph nodes $(0 / 31)$.

Six weeks postoperatively, the patient presented with increasing, painless distention of her abdomen. She was otherwise asympto-
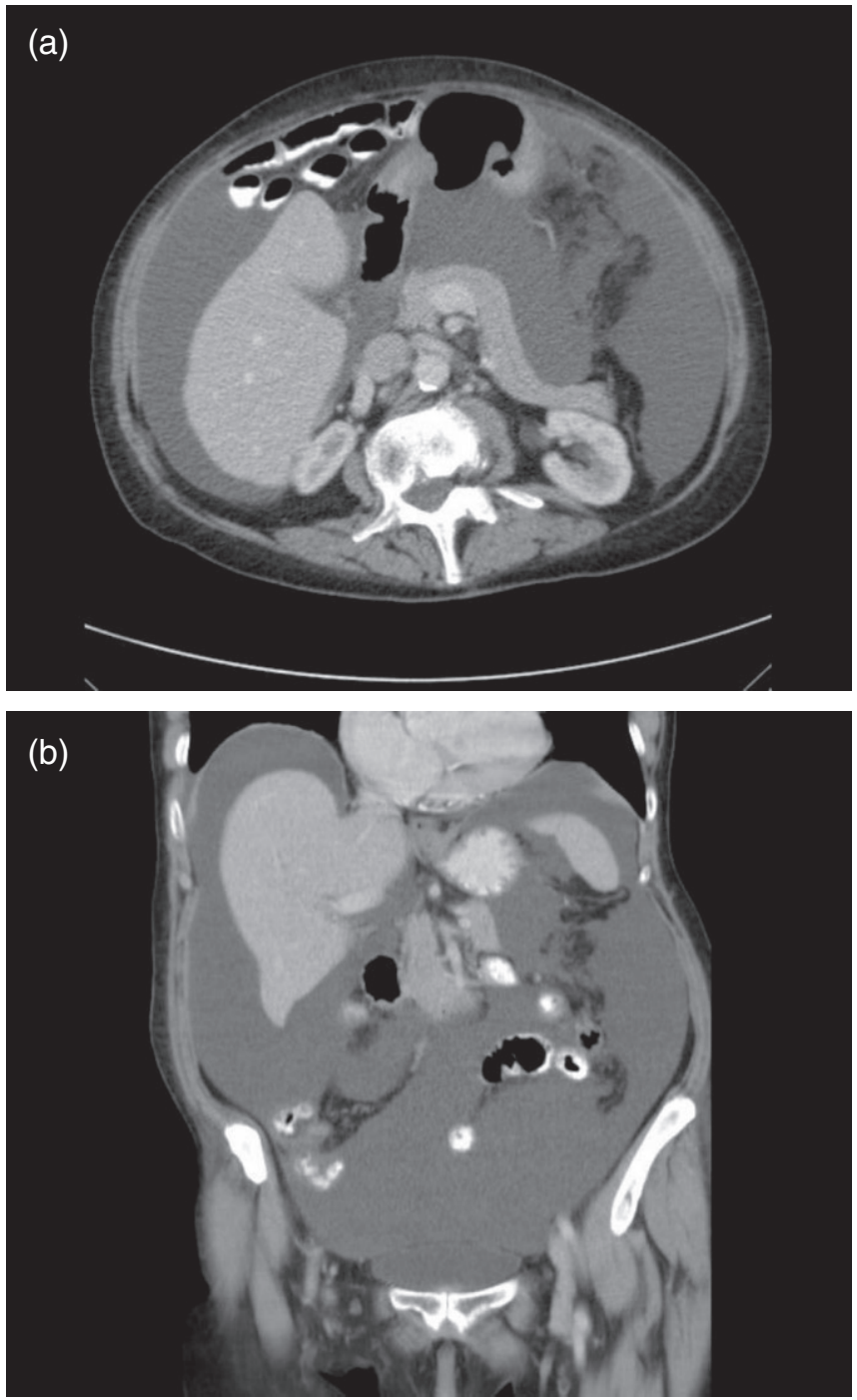

Fig. 1. (a) Axial computed tomography of the abdomen demonstrating ascites (white arrow). (b) Coronal computed tomography of the abdomen demonstrating ascites (white arrow). matic. Computed tomography revealed abdominal ascites (Fig. 1a,b). Blood tests and the carcinoembryogenic antigen levels were within normal limits.

Ultrasound guided paracentesis of the ascites and placement of a drain into the peritoneal cavity was performed. Chylous milky fluid was aspirated. A specimen was sent to the laboratory for analysis. Approximately $5 \mathrm{~L}$ of milky white opaque fluid was initially drained after insertion of the abdominal drain (Fig. 2).

The volume lost via the drain exceeded $1000 \mathrm{~mL} / 24 \mathrm{~h}$ for 2 weeks. Analysis of the fluid revealed triglyceride levels of $18.2 \mathrm{mmol} / \mathrm{L}$ (Table 1). Cytology and microbiology were negative. A diagnosis of chylous ascites was made.

The patient was commenced on a medium chain triglyceride diet. This failed to reduce the volume of fluid draining via the abdominal drain. Total parenteral nutrition (TPN) together with fasting was then instituted.

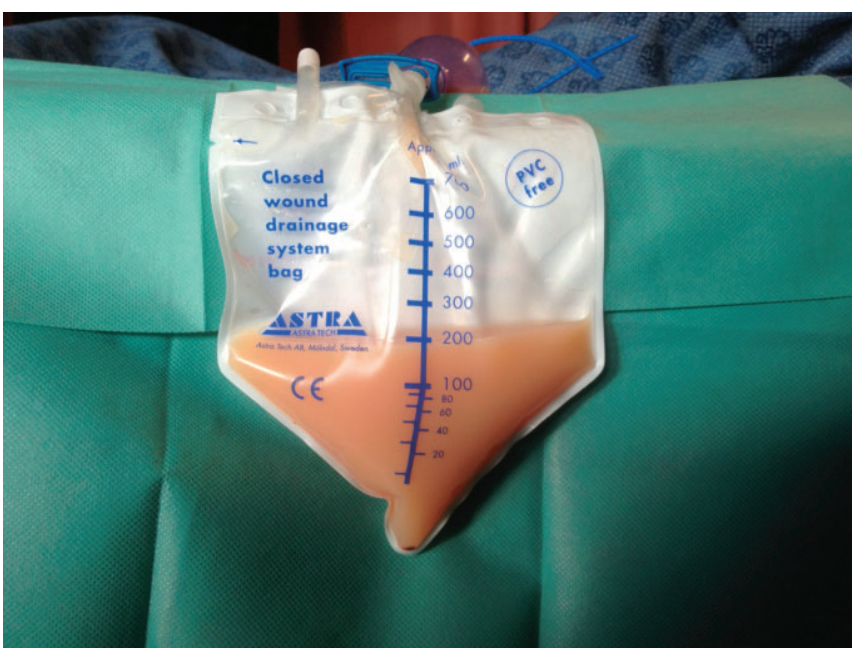

Fig. 2. Abdominal drain bag with chylous ascitic fluid.

Table 1 Peritoneal fluid

\begin{tabular}{lcc}
\hline Specimen Type: Ascitic fluid & & \\
& & \\
Triglycerides & 18.2 & $\mathrm{mmol} / \mathrm{L}$ \\
LD & 124 & $\mathrm{U} / \mathrm{L}$ \\
Protein & 66 & $\mathrm{~g} / \mathrm{L}$ \\
Albumin & 26 & $\mathrm{~g} / \mathrm{L}$ \\
Lipase & 77 & $\mathrm{U} / \mathrm{L}$ \\
Sodium & 141 & $\mathrm{mmol} / \mathrm{L}$ \\
Potassium & 3.7 & $\mathrm{mmol} / \mathrm{L}$ \\
Urea & 2.4 & $\mathrm{mmol} / \mathrm{L}$ \\
Creatinine & 58 & $\mathrm{umol} / \mathrm{L}$
\end{tabular}


The character of the fluid from the drain changed from milky white to serous after commencement of TPN. The drain was clamped. A medium chain triglyceride diet was recommenced. The patient remained well and the abdominal drain was removed on the 25 th day after insertion. The ascites did not reaccumulate.

Chylous ascites is rare and is characterized by a milky-appearing fluid containing high levels of triglycerides. ${ }^{1}$ It is due to the presence of thoracic or intestinal lymph in the abdominal cavity. ${ }^{2}$ It is an uncommon surgical complication. ${ }^{3}$ Procedures commonly implicated include oncologic thoracic and abdominal surgery and abdominal aortic surgery. ${ }^{1,3}$ Most of the data following surgery are based on anecdotal evidence and limited case reports. ${ }^{3}$ The most common symptom is progressive and painless abdominal distension. ${ }^{1}$ The most important diagnostic investigation is abdominal paracentesis and thorough analysis of the ascites including triglyceride levels, bacteriology and cytology. ${ }^{1,2}$ The triglyceride levels are typically elevated. ${ }^{2}$ Computed tomography of the abdomen may be useful. ${ }^{2}$ Lymphangiography and lymphoscintigraphy are high risk procedures that are best avoided. ${ }^{2}$ There are limited numbers of studies addressing the best treatment regimens. The management of chylous ascites is non-operative combined with repeated paracentesis and treatment of the underlying cause. ${ }^{2}$ A high-protein, low-fat diet with medium-chain triglycerides should be commenced and if this fails, strict fasting and TPN should be instituted. ${ }^{2}$ Surgery may benefit some patients who fail nonoperative management. ${ }^{2}$

Chylous ascites following anterior resection is rare, especially in the absence of neoadjuvant or adjuvant chemoradiotherapy. It can be associated with significant morbidity or even death secondary to sepsis. $^{3}$

\section{References}

1. Steinemann DC, Dindo D, Clavien PA, Nocito A. Atraumatic chylous ascites: systematic review on symptoms and causes. J. Am. Coll. Surg. 2011; 212: 899-905.

2. Cardenas A, Chopra S. Chylous ascites. Am. J. Gastroenterol. 2002; 97: 1896-900.

3. Assumpcao L, Cameron JL, Wolfgang CL et al. Incidence and management of chyle leaks following pancreatic resection: a high volume singlecentre institutional experience. J. Gastrointest. Surg. 2008; 12: 1915-23.

Kamal Galketiya, FRACS

Ian Davis, FRACS

Department of Surgery, Canberra Hospital, Canberra, Australian Capital Territory, Australia

doi: 10.1111/ans.12122 